\title{
Anesthesia for the patient undergoing total knee replacement: current status and future prospects
}

This article was published in the following Dove Press journal:

Local and Regional Anesthesia

8 March 2017

Number of times this article has been viewed

\section{Zachary A Turnbull \\ Dahniel Sastow \\ Gregory P Giambrone \\ Tiffany Tedore}

Department of Anesthesiology, Weill Cornell Medical College, New York, NY, USA
Correspondence: Zachary A Turnbull Department of Anesthesiology, Weill Cornell Medical College, 525 East 68th Street, Box 124, New York, NY 10065, USA

Tel + I 2127465643

Email ZAT2002@med.cornell.edu
Abstract: Total knee arthroplasty (TKA) has become one of the most common orthopedic surgical procedures performed nationally. As the population and surgical techniques for TKAs have evolved over time, so have the anesthesia and analgesia used for these procedures. General anesthesia has been the dominant form of anesthesia utilized for TKA in the past, but regional anesthetic techniques are on the rise. Multiple studies have shown the potential for regional anesthesia to improve patient outcomes, such as a decrease in intraoperative blood loss, length of stay, and patient mortality. Anesthesiologists are also moving toward multimodal analgesia, which includes peripheral nerve blockade, periarticular injection, and preemptive analgesia. The goal of multimodal analgesia is to improve perioperative pain control while minimizing systemic narcotic consumption. With improved postoperative pain management and rapid patient rehabilitation, new clinical pathways have been engineered to fast track patient recovery after orthopedic procedures. The aim of these clinical pathways was to improve quality of care, minimize unnecessary variations in care, and reduce cost by using streamlined procedures and protocols. The future of TKA care will be formalized clinical pathways and tracks to better optimize perioperative algorithms with regard to pain control and perioperative rehabilitation.

Keywords: TKA, regional anesthesia, analgesia

\section{Introduction}

Beginning in the early 1970s, total knee arthroplasty (TKA) was, and continues to be, a major advancement in the treatment of chronic refractory joint pain. Currently, TKA is a safe and highly effective procedure to improve mobility and patient quality of life for those suffering from end-stage osteoarthritis. ${ }^{1,2}$ Today, TKA has gained popularity and has become one of the most common orthopedic surgical procedures performed nationally, ${ }^{3,4}$ with the number of TKAs performed nationally doubling to more than 700,000 from 1999 to $2009 .{ }^{5}$ With an aging US population, the rise in TKA utilization is expected to continue..$^{5}$ As the population and surgical techniques for TKAs have evolved over time, so have the anesthetic techniques used for these procedures, resulting in an improvement in patient outcomes such as length of stay (LOS), overall cost, complication rate, perioperative pain, opioid side effects, and overall patient satisfaction. Presented here is a synopsis of the trends in TKA utilization and demographics, as well as a discussion of the current and future trends in TKA anesthesia and analgesia and the implications for patient outcomes. 


\section{Trends}

The utilization of TKA has increased significantly over time. ${ }^{3,5,6}$ In addition to an aging population, ${ }^{1,2}$ factors such as increased obesity and associated osteoarthritis have been identified as contributing to the greater need for joint replacement surgery. ${ }^{7,8}$ There also appears to be an increasing need for joint replacement in a younger patient population secondary to sports-related injuries. This may be due in part to an improved quality of the prosthetics, making these procedures reasonable early interventions. ${ }^{9}$

In a population-based cross-sectional study of Wisconsin residents during the time period 1990-2000, the age-adjusted rates of TKA increased from 162 to 294 per $100,000 .{ }^{4}$ Nationally, among Medicare enrollees, an increase of $58 \%$ was noted between 2000 and 2006 (145,242 vs 248,267, respectively). ${ }^{10,11}$ Furthermore, a study of the National Hospital Discharge Survey (NHDS) identified that primary TKA rates from 1990 to 2002 increased from 51 to 136 per 100,000 , representing a $170 \%$ increase. ${ }^{6,11}$ Additionally, during this time period, TKA revisions increased by $270 \% .{ }^{6}$ This increase in procedures is projected to continue its trajectory over time, with an estimated 3.48 million TKAs performed by the year $2030 .^{5}$

The overall cost to the health care system has mirrored the increase in the number of joint replacement procedures. In the year $2000, \sim \$ 3.2$ billion was paid by Medicare alone for hip and knee replacements. ${ }^{12}$ However, it has been argued that the increased health care cost has resulted in a lifetime societal savings of $\sim \$ 12$ billion. ${ }^{13}$

As TKA utilization has expanded over time, there have been shifts in patient demographics and outcomes as well. During the time period 1998-2008, the average LOS decreased by $\sim 1$ day, but the percentage of TKA patients who were discharged straight to their homes, as opposed to longterm or short-term care facilities, decreased from $29.1 \%$ to $25.4 \%$. Additionally, there were increases in complications such as pulmonary embolism, sepsis, nonmyocardial infarction, and pneumonia. Despite an increase in these complications, a decline of in-hospital mortality was noted over this time period, even after adjusting for the decreased LOS. During this decade, the average age of patients undergoing TKA and THA decreased by 2 to 3 years, from $\sim 69$ to 67 years and $\sim 67$ to 65 years, respectively. ${ }^{14}$ Patients aged $75-84$ years have been identified as the group with the highest overall rate when comparing 2000 to 2006 (6.6 vs 10.2 per 1,000 population, respectively); however, increases between 2000 and 2006 were identified among those aged 65-74 (5.4 vs 9.1 per 1,000 population, respectively) and $\geq 85$ years ( 2.6 vs 4.0 per 1,000 population, respectively). ${ }^{10}$ It has been projected that by 2030 , patients younger than 65 years will represent $55 \%$ to $62 \%$ of primary or revision TKAs. ${ }^{15}$

\section{Anesthetic techniques}

Over time, the anesthetic and analgesic techniques for TKA have evolved in an attempt to improve procedural outcomes as well as reduce complications such as pain and nausea, while improving patient satisfaction. ${ }^{16}$

\section{General and neuraxial anesthesia (NA)}

General anesthesia (GA) has been the dominant form of anesthesia utilized for TKA in the US; however, there has been an evolving body of literature identifying NA as well as peripheral nerve block techniques as acceptable alternatives. In general, GA has been associated with higher rates of postoperative nausea, vomiting, and delirium. NA anesthesia may be complicated by block failure or rare but devastating complications such as spinal hematoma, epidural abscess, and nerve injury. ${ }^{17}$ Furthermore, a patient's coagulation status must be understood as NA could be contraindicated in the setting of certain anticoagulant medications or bleeding disorders.

Regional anesthesia (RA) techniques for various surgical procedures have been shown to decrease pain, nausea and vomiting, and time to discharge, as well as reducing cardiovascular and pulmonary complications. ${ }^{18,19}$ A systemic review by Rodgers et al included 141 trials and 9,559 patients and found an association between NA and a decrease in deep vein thrombosis, pulmonary embolism, transfusion requirement, pneumonia, and respiratory depression. Overall mortality of patients with NA was also reduced by approximately one-third (odds ratio 0.70) compared to those who received GA..$^{20}$ A 2009 meta-analysis of 28 randomized trials involving 1,538 patients undergoing TKA did not identify sufficient evidence that anesthetic technique influenced mortality, cardiovascular morbidity, or the occurrence of deep vein thrombosis, pulmonary embolism, blood loss, or duration of surgery. ${ }^{21}$ However, RA was found to reduce postoperative pain, LOS, and facilitated rehabilitation. ${ }^{21} \mathrm{~A}$ second metaanalysis performed in 2009, including literature from 1966 to 2008 , utilized 21 randomized control trials of both THA and TKA patients for analysis..$^{22}$ The investigators found no benefit in the reduction in operating time, intraoperative blood loss, mortality, or LOS when comparing RA to GA specifically in the TKA population..$^{22}$ The study did identify a lower incidence of thromboembolic disease among the RA group; however, this finding dissipated when performing a 
subgroup analysis including only studies in which patients were given anticoagulants. ${ }^{22}$

Recent studies, utilizing large database sources that allowed for larger sample sizes and an evaluation of real world practice, have demonstrated more positive results related to the use of RA techniques for TKA. In a 2013 retrospective study, ${ }^{23} 6,030$ patients received spinal anesthesia and 8,022 patients received GA. The patients receiving spinal anesthesia had a lower rate of wound infection, blood transfusions, and overall complications. The length of surgery and hospital LOS were both decreased in the spinal anesthesia population. These effects were more pronounced among patients with numerous comorbidities. ${ }^{23}$ In a 2012 study comparing GA and NA for bilateral TKA, improved outcomes were identified in the neuraxial group. ${ }^{24}$ Of the 15,687 patients, $80.1 \%$ had GA, 13.1 had a combination of GA and NA, and 6.8 had only NA. Patients in the NA-only group required fewer blood transfusions and exhibited lower, but nonsignificant, rates of in-hospital mortality, 30-day mortality, and overall complications. ${ }^{24}$ In 2013, Memtsoudis et $\mathrm{al}^{25,26}$ published two additional studies in support of NA. In both studies, the patient population was split into three groups: NA, GA, and combined NA and GA. The first study utilized a population of 382,236 patient entries with TKA/THA and identified that patients receiving NA had significantly lower 30-day mortality rates compared to GA $(0.10 \%$ vs $0.18 \%$, respectively; $P<0.001)$ and a lower incidence of prolonged LOS, increased cost, and in-hospital complications. ${ }^{2}$ After multivariate analysis, GA was found to be associated with increased 30-day mortality (OR 1.83, 95\% CI 1.08-3.1, $P=0.02$ ), higher risk of pulmonary compromise (OR 1.83, 95\% CI, 1.43-2.35, $P<0.0001$ ), pneumonia (OR 1.27, 95\% CI 1.05-1.53, $P=0.0083$ ), all infections (OR 1.38, 95\% CI 1.26-1.52, $P<0.0001$ ), and acute renal failure (OR 1.44, 95\% CI 1.24-1.67, $P<0.0001$ ). Transfusion requirements were lowest in the NA-only group. The incidence of prolonged LOS was greatest in the GA-only group. ${ }^{25}$ The second study by Memtsoudis et $\mathrm{al}^{26}$ demonstrated benefits for NA in 30,024 sleep apnea patients undergoing TKA. Of these patients, 74\% received GA, $15 \%$ received NA and GA, and $11 \%$ received NA only with no GA. ${ }^{26}$ Rates of pulmonary, gastrointestinal, infectious, and renal complications were all lower in the NA-only patients. Transfusions, mechanical ventilation, and critical care services were lower in patients receiving NA and NA/GA compared to those receiving only GA. The GA-only patients also had the longest LOS. The cost of all three groups did not differ substantially averaging $\sim \$ 15,510$ per surgery with a standard deviation of only $\$ 225 .{ }^{26}$ The improved outcome assessment from these large database sources has been left vulnerable by the fact that these studies were retrospective and utilized administrative data sources that may be susceptible to coding errors and data quality concerns.

Despite this new recent body of evidence supporting the use of NA for TKA, the continued use of GA over RA techniques may be due to conflicting data, the resources available, and the lack of large multi-institutional trials demonstrating a positive effect.

\section{Multimodal analgesia}

Anesthesiologists are progressively moving toward multimodal analgesia regimens to combat the many mechanisms of pain and speed recovery. The goal has been to improve perioperative pain control while minimizing systemic narcotic consumption. ${ }^{27}$ This approach reduces the undesired adverse effects of narcotics such as nausea, vomiting, sedation, respiratory depression, and pruritus while increasing participation with physical activity and patient satisfaction. ${ }^{27}$ Current early recovery protocols often include pre- and postoperative opioid and nonopioid oral analgesics, as well as postoperative regional analgesic techniques in an effort to decrease the use of intravenous opioids while still providing adequate pain relief. ${ }^{27}$

\section{Peripheral nerve blockade}

There has been much investigation on optimizing peripheral nerve blocks. ${ }^{28}$ Two of the most commonly used peripheral nerve blocks for pain control after TKA are the femoral nerve block (FNB) and the adductor canal block (ACB). ${ }^{27}$ A meta-analysis was conducted to compare FNB/infusion as postoperative analgesia compared to other common analgesia modalities. ${ }^{29}$ In the first 72 hours, FNB in addition to intravenous PCA demonstrated less pain at rest and during movement, lower opioid consumption, and lower risk of nausea/vomiting, greater knee flexion, and greater patient satisfaction than those given only a PCA opioid. In contrast, when FNB was compared to the epidural group, there were no significant differences in pain during the first 72 hours after surgery as well as opioid consumption and knee flexion. Nerve catheters demonstrated less pain at rest and during movement after 24 hours and lower opioid consumption compared to a single shot nerve block. ${ }^{29}$ A noted side effect for FNBs is its effect on both sensory and motor nerves leading to quadriceps weakness and an increase in the risk of falling. ${ }^{27}$ 
Adductor canal blockade selectively blocks sensory nerves with minimal if any motor involvement. It results in a blockade of the anteromedial knee at the level of the superior pole of the patella and the medial lower leg. ${ }^{27} \mathrm{~A}$ small, double-blind, placebo controlled study was conducted to study the effects of ACB on TKA patients. ${ }^{30}$ Patients were given a continuous ACB with intermittent boluses of ropivacaine or saline. Both groups received an intravenous PCA. The ropivacaine group had significantly lower morphine consumption over the first 24 hours and lower pain upon flexion of the knee but no significant difference in pain at rest. The ropivacaine patients also successfully performed the ambulation test and the Timed Up and Go test quicker than the placebo group. ${ }^{30}$

Although numerous meta-analyses have attempted to determine whether FNB or ACB is superior, conflicting results have emerged. ${ }^{31-35}$ While some meta-analyses have found ACBs to be equivalent to FNBs in terms of pain control at rest and with movement, strength of quadriceps and adductor muscles, LOS, and incidence of nausea and vomiting, ${ }^{31}$ others have found $\mathrm{ACBs}$ to be superior. A meta-analysis by $\mathrm{Li}$ et $\mathrm{al}^{32}$ demonstrated that ACBs significantly improved Visual Analogue Scale pain scores at rest, 8 hours, and 24 hours after TKA operation compared to FNB and identified an improvement in quadriceps strength and mobility, resulting in better ambulation and faster recovery after surgery. Furthermore, meta-analyses comparing ACBs with FNB single shot block or continuous catheters demonstrated that ACBs provide equally effective analgesic control, more rapid pain relief, and decreased postoperative nausea. ${ }^{33,34}$

However, it is important to note that meta-analyses are unable to control for differences between included studies, including type of medications, concentrations, volumes, and actual location of nerve block, which could make comparisons difficult. The trend in the literature supporting ACB as a more selective sensory block, minimizing quadriceps weakness without compromising analgesia, makes ACB a preferred option for TKA.

\section{Periarticular injections (PAls)}

Seangleulur et $a l^{35}$ examined the analgesic efficacy of PAI compared to placebo/no injection in patients undergoing TKA. The PAI group had lower pain scores, opioid consumption and postoperative nausea/vomiting, higher range of motion at 24 hours, and shorter LOS than no injection or placebo. ${ }^{35}$ Again it is important to note that study variation such as type of medication used, dose, and volume can alter the efficacy of the PAI.
A study by Kelley et $\mathrm{al}^{36}$ suggested that ketorolac is the key component of PAI mixtures. In another recent study, ${ }^{37}$ an injection mixture of ropivacaine, epinephrine, ketorolac, and morphine injection was compared to FNB and sciatic nerve block with $0.5 \%$ ropivacaine. There was no significant difference in pain scores between groups. The PAI group had a shorter average LOS, but a higher narcotic consumption the day of the surgery. ${ }^{37}$ Therefore, depending on resources, a PAI can be used if RA techniques are limited.

\section{Preemptive analgesia}

Many multimodal analgesic regimens use preoperative medications in an attempt to prevent pain inflammation during the perioperative period. ${ }^{27}$ Minimizing stimulation of peripheral and central nociceptors may lead to decreases in pain postoperatively, including the development of chronic pain. ${ }^{31}$ One analgesic technique, studied in a small 48-patient randomized, double-blind, placebo-controlled trial, utilized high-dose methylprednisolone to decrease use of rescue opioids, nausea, and overall fatigue; however, sleep quality was a concern. ${ }^{38}$ Popular preemptive analgesics include acetaminophen, cyclooxygenase-2 (COX-2) inhibitors, and gabapentinoids.

A meta-analysis was conducted to analyze the effect of COX-2 inhibitors on TKA patients. Administering COX-2 inhibitors before surgery led to a decrease in postoperative pain scores, opioid consumption, pruritus, and postoperative nausea/vomiting without contributing to increases in bleeding complications. ${ }^{39}$ Furthermore, in a recent 2016 study, the COX-2 inhibitor etoricoxib was tested pre- and postoperatively and was found to decrease the amount of morphine administered in the perioperative period without any significant increase in side effects. ${ }^{40}$

Gabapentin and pregabalin are also potential options for preemptive analgesia; however, their therapeutic window has been debated. A study by YaDeau et $\mathrm{al}^{41}$ found that pregabalin, as a component of a multimodal pain regimen, did not reduce pain, increased sedation, and decreased patient satisfaction. Therefore, pregabalin was found to have no beneficial effect. A randomized, double-blind study, examining the analgesic and sedative effects of perioperative gabapentin, concluded that there was no improvement in pain control upon ambulation after 24 hours in the gabapentin group. They also noted an increase in sedation at 6 hours postoperatively with gabapentin at a dose of $1,300 \mathrm{mg} / \mathrm{d}$ relative to placebo. ${ }^{42} \mathrm{In}$ contrast to the previous studies, there are also studies showing benefits to perioperative gabapentinoids. A meta-analysis demonstrated that gabapentin was both effective in reducing postoperative 
narcotic consumption and decreasing the incidence of pruritus; however, this study was subject to potential selection bias, and further studies are indicated. ${ }^{43}$ An additional 2016 meta-analysis demonstrated inconclusive, yet suggestive, results. Hamilton et $\mathrm{al}^{44}$ analyzed numerous outcomes with regard to gabapentinoid use in TKA. Pregabalin tended to reduce postoperative pain intensity at both 24 and 48 hours but not at 72 hours and demonstrated a significant reduction in opioid consumption in the first 48 hours. Gabapentin was associated with a decrease in postoperative nausea. The study further identified an association with increased sedation postoperatively in patients receiving pregabalin. ${ }^{13}$ Some of the difficulty in determining the efficacy of gabapentinoids for preemptive analgesia may be due to the different dosing regimens used in various studies.

\section{Clinical pathways}

With improved postoperative pain management and rapid patient rehabilitation, new clinical pathways have been engineered to enhance patient recovery after orthopedic procedures. The aim of these clinical pathways is to improve quality of care, minimize unnecessary variations in care, and reduce cost by using streamlined procedures and protocols. Clinical pathways are usually specific to the procedure and hospital facility and are carried out by specialized care teams in order to produce optimal patientcentered results. ${ }^{45}$ Comprehensive preemptive multimodal analgesic regimens combined with early postoperative mobilization seem to be at the foundation of many clinical pathways. ${ }^{45-48}$ According to Total Joint Regional Anesthesia (TJRA) Clinical Pathway published by Mayo Clinic, multimodal analgesia is the cornerstone of effective therapy. All aspects of a patient's visit is optimized: first with preoperative patient education and optimization of comorbidities, followed by intraoperative multimodal analgesic regimens and the use of peripheral nerve blockade and continuous perineural catheters, and finally early postoperative interventions from a multidisciplinary Acute Pain Service and accelerated rehabilitation regimens to maximize patient outcomes. ${ }^{48}$ The pain regimen includes a combination of opioids and anti-inflammatories prior to surgery, multimodal intraoperative drug therapy such as acetaminophen and ketorolac, utilization of RA techniques such as continuous peripheral nerve catheters, and continuation of home pain therapies, if applicable. ${ }^{47}$

In a 2012 prospective study comparing a fast track protocol with a target discharge on postsurgical day 6 against standard rehabilitation, the fast track patient population had enhanced recovery based on cumulative AKSS and WOMAC index scores, reduced intake of analgesic drugs, and reduced LOS. ${ }^{45,49,50}$ Studies have also demonstrated a decrease in postoperative cognitive delirium with fast tracked patient populations, as well as a more rapid time to discharge..$^{51,52}$ Additionally, these interventions have been shown to decrease thromboembolic complications and patient readmissions. ${ }^{53}$ Additional studies have analyzed risk factors and patient demographics to enhance and optimize postoperative recovery, demonstrating that a multimodal approach is essential for a successful fast track programs, specifically in the TKA patient population. Furthermore, by decreasing LOS without compromising patient care; clinical pathways appear to be an effective cost saving metric. ${ }^{54,55}$ Although the initial data are promising, Bendetti et al ${ }^{56}$ suggest that additional larger studies are needed before a specific postoperative algorithm can be supported after TKA. Similarly, aggressive postoperative physical activity after TKA has been vital in the success of same day discharge programs after TKA operations. ${ }^{57,58}$ Gondusky et a ${ }^{57}$ highlighted their ability to discharge a cohort of 160 patients on the day of surgery, with an average age of 65 years and a mean American Society of Anesthesiology class of 1.8, to demonstrate the success of such a program. Furthermore, Schotanus et a ${ }^{58}$ demonstrated the benefits and successes of same day unicompartmental knee arthroplasty protocols to select patients in both the immediate and 3-month postoperative periods.

\section{Future and conclusion}

The need for more robust clinical outcome studies to determine best practices regarding the use of GA or RA will be needed to drive change in clinical practice. However, we will continue to see the development of clinical tracks to better optimize perioperative algorithms in regard to pain control and perioperative rehabilitation. Formalizing and perfecting clinical pathways will be the future of TKA care. Inevitably, we will observe that these pathways incorporate multidisciplinary teams, as well as multimodal analgesia methods, to maximize the patient experience and recovery. Over time, these pathways will be honed to ensure a fast and safe recovery for patients, improving patient satisfaction and ultimately postoperative outcomes. Such formalized pathways will hopefully mimic the success of enhanced recovery protocols established for other surgical patient populations.

\section{Disclosure}

The authors report no conflicts of interest in this work. 


\section{References}

1. Ethgen O, Bruyère O, Richy F, Dardennes C, Reginster J-Y. Healthrelated quality of life in total hip and total knee arthroplasty. A qualitative and systematic review of the literature. J Bone Joint Surg Am. 2004;86-A(5):963-974.

2. Harris WH, Sledge CB. Total hip and total knee replacement (1). $N$ Engl J Med. 1990;323(11):725-731.

3. Lubowitz JH, Appleby D. Cost-effectiveness analysis of the most common orthopaedic surgery procedures: knee arthroscopy and knee anterior cruciate ligament reconstruction. Arthroscopy. 2011;27(10):1317-1322.

4. Mehrotra C, Remington PL, Naimi TS, Washington W, Miller R. Trends in total knee replacement surgeries and implications for public health, 1990-2000. Public Health Rep. 2005;120(3):278-282.

5. Kurtz S, Ong K, Lau E, Mowat F, Halpern M. Projections of primary and revision hip and knee arthroplasty in the United States from 2005 to 2030. J Bone Joint Surg Am. 2007;89(4):780-785.

6. Kurtz S, Mowat F, Ong K, Chan N, Lau E, Halpern M. Prevalence of primary and revision total hip and knee arthroplasty in the United States from 1990 through 2002. J Bone Joint Surg Am. 2005;87(7):1487-1497.

7. Crowninshield RD, Rosenberg AG, Sporer SM. Changing demographics of patients with total joint replacement. Clin Orthop Relat Res. 2006;443:266-272.

8. Gillespie GN, Porteous AJ. Obesity and knee arthroplasty. Knee. 2007;14(2):81-86.

9. Losina E, Thornhill TS, Rome BN, Wright J, Katz JN. The dramatic increase in total knee replacement utilization rates in the United States cannot be fully explained by growth in population size and the obesity epidemic. J Bone Joint Surg Am. 2012;94(3):201-207.

10. Cisternas MG, Murphy L, Croft JB, Helmick CG. Racial disparities in total knee replacement among Medicare enrollees - United States, 2000-2006. MMWR Morb Mortal Wkly Rep. 2009;58(6):133-138.

11. Singh JA. Epidemiology of knee and hip arthroplasty: a systematic review. Open Orthop J. 2011;5:80-85.

12. Kane RL, Saleh KJ, Wilt TJ, et al. Total knee replacement. Evid Rep Technol Assess. 2003;86:1-80.

13. Ruiz D, Koenig L, Dall TM, et al. The direct and indirect costs to society of treatment for end-stage knee osteoarthritis. J Bone Joint Surg Am. 2013;95(16):1473-1480.

14. Kirksey M, Chiu YL, MaY, et al. Trends in in-hospital major morbidity and mortality after total joint arthroplasty: United States 1998-2008. Anesth Analg. 2012;115(2):321-327.

15. Kurtz SM, Lau E, Ong K, Zhao K, Kelly M, Bozic KJ. Future young patient demand for primary and revision joint replacement: national projections from 2010 to 2030. Clin Orthop Relat Res. 2009;467(10):2606-2612.

16. Macario L, Weinger M, Carney S, Kim AB. Which clinical anesthesia outcomes are important to avoid? The perspective of patients. Anesth Analg. 1999;89(3):652-658.

17. Horlocker TT. Complications of regional anesthesia and acute pain management. Anesthesiol Clin. 2011;29(2):257-278

18. Hadzic A, Williams BA, Karaca PE, et al. For outpatient rotator cuff surgery, nerve block anesthesia provides superior same-day recovery over general anesthesia. Anesthesiology. 2005;102(5):1001-1007.

19. Ilfred BM, Vandenborne K, Duncan PW, et al. Ambulatory continuous interscalene nerve blocks decrease the time to discharge readiness after total shoulder arthroplasty. Anesthesiology. 2006;105(5):999-1007.

20. Rodgers A, Walker N, Schug S, et al. Reduction of postoperative mortality and morbidity with epidural or spinal anaesthesia: results from overview of randomised trials. BMJ. 2000;321(7275):1493.

21. Macfarlane AJR, Prasad GA, Chan VWS, Brull R. Does regional anesthesia improve outcome after total knee arthroplasty? Clin Orthop Relat Res. 2009;467(9):2379-2402.

22. Hu S, Zhang Z-Y, Hua Y-Q, Li J, Cai Z-D. A comparison of regional and general anaesthesia for total replacement of the hip or knee: a meta-analysis. J Bone Joint Surg Br. 2009;91(7):935-942.
23. Pugely AJ, Martin CT, Gao Y, Mendoza-Lattes S, Callaghan JJ. Differences in short-term complications between spinal and general anesthesia for primary total knee arthroplasty. J Bone Joint Surg Am. 2013;95(3): 193-199.

24. Stundner O, Chiu Y-L, Sun X, et al. Comparative perioperative outcomes associated with neuraxial versus general anesthesia for simultaneous bilateral total knee arthroplasty. Reg Anesth Pain Med. 2012;37(6): 638-644.

25. Memtsoudis SG, Sun X, Chiu Y-L, et al. Perioperative comparative effectiveness of anesthetic technique in orthopedic patients. Anesthesiology. 2013;118(5):1046-1058.

26. Memtsoudis SG, Stundner O, Rasul R, et al. Sleep apnea and total joint arthroplasty under various types of anesthesia: a population-based study of perioperative outcomes. Reg Anesth Pain Med. 2013;38(4):274-281.

27. Moucha CS, Weiser MC, Levin EJ. Current strategies in anesthesia and analgesia for total knee arthroplasty. J Am Acad Orthop Surg. 2016; 24(2):60-73.

28. Bendtsen TF, Moriggl B, Chan V, Borglum J. The optimal analgesic block for total knee arthroplasty. Reg Anesth Pain Med. 2016;41:711-719.

29. Chan EY, Fransen M, Parker DA, Assam PN, Chua N. Femoral nerve blocks for acute postoperative pain after knee replacement surgery. Cochrane Database Syst Rev. 2014;(5):CD009941.

30. Jenstrup MT, Jæger P, Lund J, et al. Effects of adductor-canal-blockade on pain and ambulation after total knee arthroplasty: a randomized study. Acta Anaesthesiol Scand. 2012;56(3):357-364.

31. Dong C-C, Dong S-L, He F-C. Comparison of adductor canal block and femoral nerve block for postoperative pain in total knee arthroplasty: a systematic review and meta-analysis. Medicine (Baltimore). 2016;95(12):e2983.

32. Li D, Yang Z, Xie X, Zhao J, Kang P. Adductor canal block provides better performance after total knee arthroplasty compared with femoral nerve block: a systematic review and meta-analysis. Int Orthop. 2016;40(5):925-933.

33. Kuang M-J, Xu L-Y, Ma J-X, et al. Adductor canal block versus continuous femoral nerve block in primary total knee arthroplasty: a meta-analysis. Int J Surg. 2016;31:17-24.

34. Gao F, Ma J, Sun W, Guo W, Li Z, Wang W. Adductor canal block versus femoral nerve block for analgesia after total knee arthroplasty: a systematic review and meta-analysis. Clin J Pain. Epub 2016 Jun 17.

35. Seangleulur A, Vanasbodeekul P, Prapaitrakool S, et al. The efficacy of local infiltration analgesia in the early postoperative period after total knee arthroplasty: a systematic review and meta-analysis. Eur $J$ Anaesthesiol. 2016;33(11):816-831.

36. Kelley TC, Adams MJ, Mulliken BD, Dalury DF. Efficacy of multimodal perioperative analgesia protocol with periarticular medication injection in total knee arthroplasty: a randomized, double-blinded study. J Arthroplasty. 2013;28(8):1274-1277.

37. Spangehl MJ, Clarke HD, Hentz JG, Misra L, Blocher JL, Seamans DP. The Chitranjan Ranawat Award: periarticular injections and femoral \& sciatic blocks provide similar pain relief after TKA: a randomized clinical trial. Clin Orthop Relat Res. 2015;473(1):45-53.

38. Lunn TH, Kirstensen BB, Andersen LO, et al. Effect of high-dose preoperative methylprednisolone on pain and recovery after total knee arthroplasty: a randomized, placebo-controlled trial. Br J Anaesth. 2010; 106(2):230-238.

39. Lin J, Zhang L, Yang H. Perioperative administration of selective cyclooxygenase- 2 inhibitors for postoperative pain management in patients after total knee arthroplasty. J Arthroplasty. 2013;28(2):207-213.

40. Munteanu AM, Florescu SC, Anastase DM, Stoica CI. Is there any analgesic benefit from preoperative vs. postoperative administration of etoricoxib in total knee arthroplasty under spinal anaesthesia? A randomised double-blind placebo-controlled trial. Eur J Anaesthesiol. 2016;33(11):840-845.

41. YaDeau JT, Lin Y, Mayman DJ, et al. Pregabalin and pain after total knee arthroplasty: a double-blind, randomized, placebo-controlled, multidose trial. Br J Anaesth. 2015;115(2):285-293. 
42. Lunn TH, Husted H, Laursen MB, Hansen LT, Kehlet H. Analgesic and sedative effects of perioperative gabapentin in total knee arthroplasty; a randomized, double-blind, placebo-controlled dose-finding study. Pain . 2015;156(12):2438-2448.

43. Han C, Li X-D, Jiang H-Q, Ma J-X, Ma X-L. The use of gabapentin in the management of postoperative pain after total knee arthroplasty: a PRISMA-compliant meta-analysis of randomized controlled trials. Medicine (Baltimore). 2016;95(23):e3883.

44. Hamilton TW, Strickland LH, Pandit HG. A meta-analysis on the use of gabapentinoids for the treatment of acute postoperative pain following total knee arthroplasty. J Bone Joint Surg Am . 2016;98(16):1340-1350.

45. den Hertog A, Gliesche K, Timm J, Mühlbauer B, Zebrowski S. Pathway-controlled fast-track rehabilitation after total knee arthroplasty: a randomized prospective clinical study evaluating the recovery pattern, drug consumption, and length of stay. Arch Orthop Trauma Surg. 2012;132(8):1153-1163.

46. Husted H, Hansen HC, Holm G, et al. What determines length of stay after total hip and knee arthroplasty? A Nationwide Study in Denmark. Arch Orthop Trauma Surg. 2009;130(2):263-268.

47. Hebl JR, Dilger JA, Byer DE, et al. A pre-emptive multimodal pathway featuring peripheral nerve block improves perioperative outcomes after major orthopedic surgery. Reg Anesth Pain Med. 2008;33(6): 510-551.

48. Duncan CM, Moeschler SM, Horlocker TT, Hanssen AD, Hebl JR. A self-paired comparison of perioperative outcomes before and after implementation of a clinical pathway in patients undergoing total knee arthroplasty. Reg Anesth Pain Med. 2013;38(6):533-538.
49. Liow RY, Walker K, Wajid MA, Bedi G, Lennox CM. The reliability of the American Knee Society Score. Acta Orthop Scand. 2000;71(6): 603-608.

50. Davies AP. Rating systems for total knee replacement. Knee. 2002; 9(4):261-266.

51. Krenk L, Kehlet H, Bæk Hansen T, Solgaard S, Soballe K, Rasmussen LS. Cognitive dysfunction after fast-track hip and knee replacement. Anesth Analg. 2014;118(5):1034-1040.

52. Van Den Eeden YN, De Turck BJ, Van Den Eeden FM. 24hours stay after hip replacement. Acta Orthop. 2016;88(1):24-28.

53. Pennington JM, Jones DPG, McIntyre S. Clinical pathways in total knee arthroplasty: a New Zealand experience. J Orthop Surg (Hong Kong) 2003;11(2):166-173.

54. Rodriguez-Merchan EC. Pros and cons of fast-track total knee arthroplasty. Int J Orthop. 2015;2(3):270-279.

55. Kim S, Losina E, Solomon DH, Wright J, Katz JN. Effectiveness of clinical pathways for total knee and total hip arthroplasty: literature review. J Arthroplasty. 2003;18(1):69-74.

56. Bendetti MG, Sarti D, Bonfiglioli Stagni S, Mariani E. Setting, clinical pathways, fast track and rehabilitation following primary knee arthroplasty: a literature review. Open Rehabil J. 2015;8(1):17-24.

57. Gondusky JS, Choi L, Khalaf N, Patel J, Barnett S, Gorab R. Day of surgery discharge after unicompartmental knee arthroplasty: an effective perioperative pathway. J Arthroplasty. 2014;29(3):516-519.

58. Schotanus MG, Bemelmans YF, Grimm B, Heyligers IC, Kort NP. Physical activity after outpatient surgery and enhanced recovery for total knee arthroplasty. Knee Surg Sports Traumatol Arthrosc. Epub 2016 Aug 4:1-6.
Local and Regional Anesthesia

\section{Publish your work in this journal}

Local and Regional Anesthesia is an international, peer-reviewed, open access journal publishing on the development, pharmacology, delivery and targeting and clinical use of local and regional anesthetics and analgesics. The journal is included in PubMed, and welcomes submitted papers covering original research, basic science, clinical studies,

\section{Dovepress}

reviews and evaluations, guidelines, expert opinion and commentary, case reports and extended reports. The manuscript management system is completely online and includes a very quick and fair peer-review system, which is all easy to use. Visit http://www.dovepress.com/ testimonials.php to read real quotes from published authors. 